Research Article

\title{
Research on Higher Education Evaluation and Decision-Making Based on Data Mining
}

\author{
Liu Feng \\ School of Marxism, Shaoyang University, Shaoyang 422000, China \\ Correspondence should be addressed to Liu Feng; 2901@hnsyu.edu.cn
}

Received 12 October 2021; Revised 26 October 2021; Accepted 26 October 2021; Published 8 November 2021

Academic Editor: Rahman Ali

Copyright (c) 2021 Liu Feng. This is an open access article distributed under the Creative Commons Attribution License, which permits unrestricted use, distribution, and reproduction in any medium, provided the original work is properly cited.

\begin{abstract}
Educational data mining is concerned with developing methods to explore the data from educational environments which provides insights that help in understanding the learning process and improving the educational outcomes. The evaluation and decision-making methods of higher education resources ignore the number of specific basic systems of resource evaluation and decision-making, resulting in the low accuracy of evaluation and decision-making. Therefore, a research on higher education evaluation and decision-making based on data mining is proposed. We analyze the application of big data in the field of higher education and design its optimal curriculum design model. We calculate the phased teaching task objectives of higher education curriculum, form its curriculum teaching guidance according to the influence degree between learners' learning progress and learners' thinking limitations, and obtain the learning effect produced by the optimal selection of curriculum teaching content. Then the probability of learners completing the structured teaching goal is calculated, so as to establish the optimal curriculum design model of higher education. Finally, we obtain the quantitative values of different experiences, extract the main influencing factors of resource evaluation and decision-making, and carry out higher education resource evaluation and decision-making analysis on this basis. The experimental results show that the research method improves the flexibility and universal applicability of higher education evaluation and decision-making, achieving an evaluation accuracy of above $90 \%$ and with below $7 \%$ error rate.
\end{abstract}

\section{Introduction}

At present, high-quality economic and social development is inseparable from the innovative development of higher education. As the fundamental driving force of national and social development, high-quality talents affect the country's international status and long-term development strategy $[1,2]$. Each local higher education institution has the basic functions of talent training and social service, but most colleges and universities have different positioning, levels, and teaching priorities, so it is difficult to meet the requirements of innovative development of higher education [3]. In China, the development and reform in the field of education are under the heavy pressure of great challenges. The combination of education and data mining has become an inevitable trend in the development of education. In the whole process of educational activities, all data related to educational activities and the collection of data used to create potential value for educational development are collectively referred to as educational big data [4]. Under the current research situation in the field of education at home and abroad, from a strategic perspective, education big data can be positioned as a scientific help to promote the transformation of new strategic assets in education and drive the comprehensive reform in the field of education and the basis for the development of intelligent education. The application value of education big data should be reflected in the deep integration with the mainstream business of education and the continuous promotion of intelligent reform of the education system, specifically in improving scientific education management, promoting the reform process of education and teaching mode, guiding the practice of personalized learning and teaching activities, promoting the improvement of education and teaching evaluation system, driving the transformation of scientific research paradigm, and leading the humanized education service system suitable 
for people. However, due to the uniqueness and social complexity of the education field, the development of education big data in China also faces many difficult challenges, such as the full implementation, information security of education and teaching data, and data operation and maintenance and governance. Only the education department which attaches importance to and deeply studies and solves these problems can promote the vigorous development of education big data in China's education field.

Data mining in education is an emerging field that is concerned with developing methods to explore the types of data that come from the educational environment. The research in this field is important because it helps to better understand the learning process of students and in improving the educational outcomes [5]. Educational data mining researchers have been addressing the questions of cognition, motivation, social discourse, affect, and language, etc., using data from educational environments [6]. Therefore, this study focuses on the use of intelligent data mining techniques for education evaluation and decisionmaking. There are a number of relevant studies focusing on the use of ML in educational evaluation and decisionmaking. Some of them are discussed as follows.

Reference [7] proposed an intelligent decision support system to predict students' performance in advance. They identify the features that influence students' performance and develop an accurate prediction model for predicting the performance of the students. A subjective method is used for identification of academic and socioeconomic features and then a decision tree-based model is adopted to learn the relationship between the features and academic grades. Reference [8] proposes to use machine learning algorithm to support academic decision-making of higher education institutions and use machine learning algorithm to predict graduation rate in a real case study to support decisionmaking. Using confusion matrix and receiver operating characteristic curve, support vector machine and artificial neural network are compared, and the algorithm method and architecture are given. Real data from five undergraduate engineering projects at Columbia Regional University illustrate the results of the study. In view of the challenges faced by educational decision-making in the era of big data, reference [9] proposed school management information system. Despite the benefits of school management information system (SMIS), the concept of data-driven school culture has not been realized in many educational institutions. The challenge of data quality in the era of big data has hindered many schools from realizing the real potential of SMI. This paper analyzes the use, characteristics, and inhibitory factors of SMIS. A five-stage conceptual model is proposed to help managers make timely and high-quality decisions, enrich the theoretical landscape of SMI in the era of big data, and lay a foundation for the establishment of educational decision-making model. Although the above research has made some progress, there is still something worth exploring for educational evaluation and decisionmaking. Therefore, this paper puts forward the research on higher education evaluation and decision-making based on data mining. Educational evaluation and decision-making are related to the sustainable development of education. The educational evaluation and decision-making scheme with scientific basis is of far-reaching significance not only in theoretical issues, but also in practical implementation.

We propose a higher education evaluation and decisionmaking system based on data mining. We first calculate the phased teaching task objectives of higher education curriculum and form its curriculum teaching guidance according to the influence degree between learners' learning progress and learners' thinking limitations. Then we obtain the learning effect produced by the optimal selection of curriculum teaching content and the probability of learners completing the structured teaching goal is calculated, so as to establish the optimal curriculum design model of higher education. Finally, the quantitative values of different experiences are obtained, and the main influencing factors of resource evaluation and decision-making are extracted to carry out the higher education resource evaluation and decision-making analysis.

The rest of the paper is organized as follows. Section 2 discusses the big data that comes from the educational environment. In Section 3, we talk about the intelligent evaluation of the data that come from educational environments. In Section 4, we discuss evaluation and decision-making of higher education resources. Section 5 presents the experimental analysis and Section 6 is the conclusion of our work.

\section{Application of Big Data in Higher Education}

Big data refers to a dataset that cannot be captured, managed, and processed by conventional software tools within a certain time range. It is a massive, high growth rate and diversified information asset that needs to update the processing mode to have stronger decision-making power, insight, and discovery power and process optimization ability. Big data does not use such shortcuts as sampling survey or probability analysis but analyzes and studies all data, that is, sample = population. "Big data" requires a new processing mode to have stronger decision-making power, insight, and discovery power and process optimization ability to adapt to massive, high growth rate and diversified information assets. It is a dataset with a large scale that greatly exceeds the capacity of traditional database software tools in terms of acquisition, storage, management, and analysis of fast data flow, diverse data types, and low value density.

According to the research subject of education big data, education big data can be divided into broad education big data and narrow education big data. Broad education big data refers to all behavior data in the daily education and teaching practice of educational institutions, and narrow education big data refers to learner behavior data $[10,11]$. Education big data refers to the collection of all data generated in the whole education and teaching activities, collected according to the needs of education and teaching, conducive to the development of education and teaching and of potential value.

Education big data is a collection of a large amount of data generated in the field of education. It is a collection 
containing the data of the whole education process. It is a multitype, multidimensional, and multiform data collection oriented to specific education topics that supports educational decision-making and personalized learning through data mining and learning analysis.

Under the influence of data mining, data mining and analysis have become the most dependent means of educational management. The previous intuition and experience model will be gradually abandoned. The scientific management of educational big data based on comprehensive data mining and analysis will replace the intuitive experience management model. With the continuous progress and rapid development of technology, educational data mining and analysis will continue to deepen. It can not only mine the existing relationship between certain data, but also explore and analyze the unknown and uncertain causal relationship hidden between data. The deep application of data mining in the field of education can widely mine and analyze educational data, analyze the existing data association and the potential value hidden behind the data, find the deficiencies and defects in educational and teaching activities, put forward solutions, and optimize educational management schemes. Data mining can be used to collect the data generated by daily educational activities, store and maintain these data, or establish models to statistically analyze the personnel data, educational resource data, and daily teaching data of educational institutions, so as to extract useful information and provide data support for scientific decisionmaking for educational and teaching activities.

With the first mock exam application in the field of education, the educational assessment activity is changing from the previous intuitive experience mode to a more convincing data analysis mode, from the overall direction assessment to the individual full aspect assessment, from the single mode assessment to the comprehensive mode analysis and evaluation. The transformation of educational evaluation activities is not only reflected in the evaluation idea. With the support of data mining, the evaluation object of educational evaluation is expanded from the original evaluation of students to the evaluation of educational activities and teaching quality, and the teaching evaluation activities are no longer completed only by students' examination results and teaching activity discipline. Through multidimensional, multilevel, and diversified educational evaluation, we can realize the fairness and justice of educational evaluation, optimize educational and teaching activities, and guide the work of educational and teaching activities.

Educational big data abandons the traditional mode of pursuing unilateral causality; instead it pursues complex and multifaceted correlations between data and then displays it in front of users in a concise and clear manner in the form of intuitive graphics and images. The transformation of this analysis mode is an indispensable change to promote the innovative development of education. In the era of educational big data, educational scientific research abandons the traditional random sampling analysis to seek the causal relationship of data and then changes to statistical analysis of all data to find the correlation between data, which makes the research on education and teaching be carried out in a more comprehensive, detailed, and scientific way.

\section{Research on Intelligent Evaluation of Education Big Data}

As one of the main research issues in the field of education, educational evaluation plays a vital role in the direction of educational reform and management decision-making. However, most of the current education evaluation indicators are formulated with reference to the relevant evaluation index systems at home and abroad and combined with the experience questionnaire in practical operation. It is difficult to judge the relationship, importance, and rationality of the indicators. Applying association rules and rough set theory to each evaluation system can sort and reduce the indexes, optimize the evaluation indexes to a certain extent, and find a reasonable and simple evaluation index system. It is very meaningful to apply data mining to teaching evaluation and explore the relationship between education index data, so as to establish an education and teaching evaluation system.

\subsection{Optimal Curriculum Design Model of Higher Education.} In the process of establishing the optimal curriculum design model of higher education, we first obtain the teaching resource needs of learners in different environments, determine the teaching task objectives in higher education, conduct digital evaluation and decision-making on higher education learning resources, and give the presentation characteristics of higher education curriculum content [12], Decompose and reorganize the knowledge content of higher education courses according to the needs of network teaching environment and the teaching objectives of network courses, provide a learning curriculum design structure suitable for learners' higher education teaching and learning mode, and obtain learners' internal psychological activity process and cognitive law [13], so as to establish the optimal curriculum design model of higher education. The specific process is as follows.

Assuming that $L(\delta)$ represents the higher education learning needs of learners in different environments, $p(c)$ represents the online course content, and $\lambda(o)$ represents the task activity needs in higher education, the teaching task objectives in higher education are determined by the following formula:

$$
\theta(e)=\frac{L(\delta) \times p(c)}{\varpi(b)} \times \eta^{\tau}(w),
$$

where $₫(b)$ represents the well-structured field of teaching knowledge provided by the higher education network.

Assuming that $g(U)$ represents the online course content design process of higher education and $g(U)$ represents the teaching activities of learners in different environments, through the digital evaluation and decision-making of higher education learning resources [14], the performance characteristics of higher education course content are given by

$$
\psi(\kappa)=\frac{g(U) \times \mu(W)}{v(o)} \times \mu(j) \times \omega(b),
$$


where $v(o)$ and $\mu(j)$ represent the teaching content performance characteristics of learners' linear learning and jump learning, respectively, decompose and reorganize the knowledge content $\xi(c)$ represented by higher education courses according to the needs of network teaching environment and the teaching objectives of network courses, and use formula (3) according to the organizational form of higher education learners. Provide a learning curriculum design structure suitable for learners' teaching and learning mode of higher education:

$$
\chi(y)=\frac{v(o) \times \mu(j)}{\xi(c)} \times \frac{k(\rho) \times z(i)}{\ell(a) / \partial(n)},
$$

where $k(\rho)$ represents the learner's learning motivation and BB represents the learner's performance characteristics in the learning process. Then, the learner's internal psychological activity process $\mathrm{CC}$ and cognitive law DD are obtained by

$$
\left\{\begin{array}{l}
\ell(a)=\frac{\zeta(c) \times \gamma(l)}{v(o)} \iota(\vartheta), \\
\partial(n)=\frac{\iota(\vartheta) \times \mu(j)}{L(\delta)},
\end{array}\right.
$$

where $\iota(\vartheta)$ represents the learning status of learners in the whole distance teaching process and $\gamma(l)$ represents the optimization selection process of higher education teachers for educational curriculum content. The optimal curriculum design model of higher education is established by using the following formula:

$$
m(g)=\frac{\iota(\vartheta)}{B(i)} \times \varsigma(\Omega) \times \alpha(d),
$$

where $B(i)$ represents the learner's autonomous learning strategy provided in the optimal curriculum design of higher education, $\varsigma(\Omega)$ represents the learners' attention to the teaching content of the optimal curriculum of higher education [1], and $\alpha(d)$ represents the satisfaction of the optimal curriculum content of higher education to the learning needs of different types of learners.

\subsection{Optimal Curriculum Design Model of Higher Education Based on Data Mining}

3.2.1. Update of Higher Education Curriculum Design Process. In the process of establishing the optimal curriculum design model of higher education, first obtain the ideology of higher education curriculum design, calculate the phased teaching task objectives of higher education curriculum, and form its higher education curriculum teaching guidance according to the influence degree between learners' learning progress and learners' thinking limitations. The learning effect of the optimal selection of higher education curriculum teaching content at a certain stage is obtained [15], and the probability of learners completing the structured teaching goal of higher education optimal curriculum design under this learning effect is given. Therefore, the higher education curriculum design process is updated. The specific process is as follows.
Assuming that $h(t)$ represents learning resources that conform to the cognitive laws of different types of learners and $t(e)$ represents learners' learning enthusiasm, the ideology of higher education curriculum design is obtained by the following formula:

$$
I_{h}^{t+1}=I_{h}^{t}+r \times I_{h}^{t}\left(\frac{1-I_{h}^{t}}{K}\right) h(t)+\frac{\left(R_{1}-R_{2}\right)}{2 t(e)},
$$

where $r$ represents the different essential attributes of various higher education courses, $K$ represents the improvement rate of higher education quality, $R_{1}$ and $R_{2}$, respectively, represent the capacity of distance curriculum teaching resources, and the higher education curriculum design ideology establishes the higher education curriculum teaching goal $S_{h}^{t}$ on the basis of meeting the students' autonomous learning needs and the clarity of the real society; the phased teaching task objectives of higher education courses are calculated using the following equation:

$$
S_{h}^{t}=c_{1} \times\left|I_{h}^{t}-V_{h}^{t}\right|+c_{2} \times\left|I_{h}^{t}-P_{h}^{t}\right|
$$

where $c_{1}$ and $c_{2}$, respectively, represent the learning direction of learners and the weight of teaching priorities in higher education courses, $V_{h}^{t}$ represents the characteristics of higher education disciplines, the matching degree between the needs of distance teaching environment and the teaching objectives of higher education courses, which constitutes the teaching content of higher education courses, and $P_{h}^{t}$ represents the limitation of learners' thinking breaking through abstract logical thinking. The degree of influence between learners' learning progress and learners' thinking limitations constitutes the teaching orientation of higher education courses, which is calculated by the following formula:

$$
P_{h}^{t}=\frac{\sum_{k \in \Omega(h)} I_{k}^{t}+\sum_{t \in(\Omega-\Omega(h))} V_{r}^{t}}{\delta(c)},
$$

where $\Omega(h)$ represents the performance of learner $h$ in the learning process of higher education courses and $G_{h}^{t}$ represents the task and activity needs of online courses, expressed as follows:

$$
\left\{\begin{array}{l}
G_{h}^{t}=w_{e} \times E_{e v}+w_{e} \times E_{e d}+w_{t} \times E_{t}+w_{p} \times E_{p}+w_{c} \times E_{c}, \\
w_{e v i}+w_{e d u}+w_{t r a}+w_{p r i}+w_{c o n}=1,
\end{array}\right.
$$

where $E_{e v}, E_{e d}, E_{t}, E_{p}$, and $E_{c}$, respectively, represent the online course demand, learners' psychological characteristics, learning motivation, learning interest, and the selection form of higher education course learning content and $w_{\text {evi }}$, $w_{e d u}, w_{\text {tra }}, w_{\text {pri }}$, and $w_{\text {con }}$, respectively, represent learners' preference coefficient for higher education course task activities [16]; learning effect of the optimized selection of teaching content of higher education courses at a certain stage is given by

$$
U_{h}^{t}=w_{g} \times G_{h}^{t}+w_{s} \times\left(1-S_{h}^{t}\right)+\varepsilon_{h}^{t},
$$

where $w_{g}$ and $w_{s}$ represent the weights of synchronous teaching and asynchronous teaching of higher education, 
respectively, and the learner's willingness to learn independently is expressed as follows:

$$
\begin{aligned}
& A W_{h}^{t}=U_{b}^{t}-U_{h}^{t} ; \\
& A W_{h}^{t} \begin{cases}\geq W T, & \text { Move, } \\
<W T, & \text { Stay, }\end{cases}
\end{aligned}
$$

where $U_{b}^{t}$ represents the optimal learning effect of learners in the process of optimal curriculum design of higher education, $A W_{h}^{t}$ represents learners' willingness to learn independently, and $W T$ represents the threshold of learners' willingness to learn independently. When learners' willingness to learn $A W_{h}^{t}$ is greater than or equal to the threshold $\mathrm{EE}$, learners can effectively complete homework submission directly related to learning to ensure learners' learning quality $U_{h}^{t}$; the probability of learners completing the structured teaching goal $L_{i j}$ of higher education curriculum design is calculated as follows:

$$
P_{i j}^{t}=\frac{\exp \left(U_{h \longrightarrow i j}^{t}\right)}{\sum \exp \left(U_{h \longrightarrow x y}^{t}\right)},
$$

where $\sum \exp \left(U_{h \rightarrow x y}^{t}\right)$ represents that learners choose higher education curriculum content according to individual learning needs and follow the maximization of utility at each stage of higher education curriculum teaching implementation.

Based on the calculation results of formula (12), according to different learners and the whole learning community, provide appropriate learning strategies for higher education courses to help learners complete teaching tasks and objectives and then use formula (13) to update the higher education course design process:

$$
V_{i j}^{t+1}=a(1+R) V_{i j}^{t}+(1+a)\left[\frac{\left(I_{h}^{t}+\sum_{m \in \Omega(i \bar{j})} V_{m}^{t}\right)}{\delta(c)}\right] .
$$

In the formula, $a$ represents the weight of distance teaching content, $R$ represents the network content teaching form selected by learners at the current stage, $I_{t}$ represents the correlation between the design of higher education activity strategy and teaching process, and $\Omega(i \bar{j})$ represents the change of teaching activity organization form. If learners complete postschool practice, self-test, and evaluation of higher education courses, use formula (14) to update the content of higher education curriculum design:

$$
V_{i j}^{t+1}=a(1-D) V_{i j}^{t}+(1-a)\left[\frac{\left(\sum_{m \in \Omega_{i j}} V_{m}^{t}\right)}{\delta(c)}\right],
$$

where $D$ represents the quality of higher education curriculum design, $\Omega_{i j}$ represents the improvement of learners' learning quality, and $\delta(c)$ represents learners' phased learning performance.

3.2.2. Establishing the Optimal Curriculum Design Model of Higher Education. In the process of establishing the optimal curriculum design model of higher education, based on the updated results of the higher education curriculum design process and combined with the multiattribute decisionmaking theory, the initial optimal curriculum decision matrix of higher education is established to obtain the organization form of the operation unit of the optimal curriculum design of higher education and the organization form of the knowledge branch of higher education [17]. On this basis, the optimal curriculum design model of higher education is established. The specific process is as follows.

Assuming that $\tilde{x}_{i j}=\left[x_{i j}^{1}, x_{i j}^{2}\right]$ represents the number of weight intervals given by learners' evaluation index $B_{j}$ of higher education curriculum design scheme $A_{i}$, the initial optimal curriculum decision matrix of higher education is established as follows:

$$
\tilde{X}_{i j}=\left(\tilde{x}_{i j}\right)_{m \times n}:\left\{\begin{array}{ccc}
\tilde{x}_{11} & \cdots & \tilde{x}_{1 n} \\
& \vdots \\
\tilde{x}_{m 1} & \cdots & \tilde{x}_{m n}
\end{array}\right\} .
$$

Assuming that $\Omega$ is a set of mathematical forms with incomplete attribute weight information of higher education courses, the attribute weight value $\left(\omega_{1}^{T}, \omega_{2}^{T}, \ldots, \omega_{n}^{T}\right)$ when the model is optimal is obtained in combination with the coefficient theory. According to the learners' own weight and each learner's digestion of the teaching content of the optimal course of higher education, the weighted average operator is used to form the comprehensive attribute value for the optimal selection of each higher education course:

$$
\varphi_{i}=\sum_{k=1}^{d} \sum_{j=1}^{n}\left[f_{i j}^{(k)}\left(t_{k}\right) \omega_{j}^{T}\right] \lambda_{k} .
$$

Bring in the weight value $\left(\omega_{1}^{T}, \omega_{2}^{T}, \ldots, \omega_{n}^{T}\right)$ calculated by formula (16), and then sort the comprehensive decisionmaking attribute values of each higher education course to obtain the optimal course of higher education, and establish the optimal course design model of higher education, as shown in Figure 1.

\section{Evaluation and Decision-Making of Higher Education Resources}

4.1. Influencing Factors of Higher Education Resource Evaluation and Decision-Making. In the process of analyzing the evaluation and decision-making of higher education resources, firstly, based on the cognitive theory, it is obtained that there are certain different quantitative values in the evaluation and decision-making of educational resources when college students face the evaluation and decisionmaking of educational resources due to the differences, and the main influencing factors of resource evaluation and decision-making when different college students face the evaluation and decision-making of educational resources are calculated. The specific process is as follows.

It is assumed that $s$ represents the impact factors of various conflict events on higher education resource evaluation and decision-making, $x$ represents the abnormal measurement value of higher education resource evaluation and decision-making, $n$ represents the time limit of higher education resource evaluation and decision-making, and $j$ 


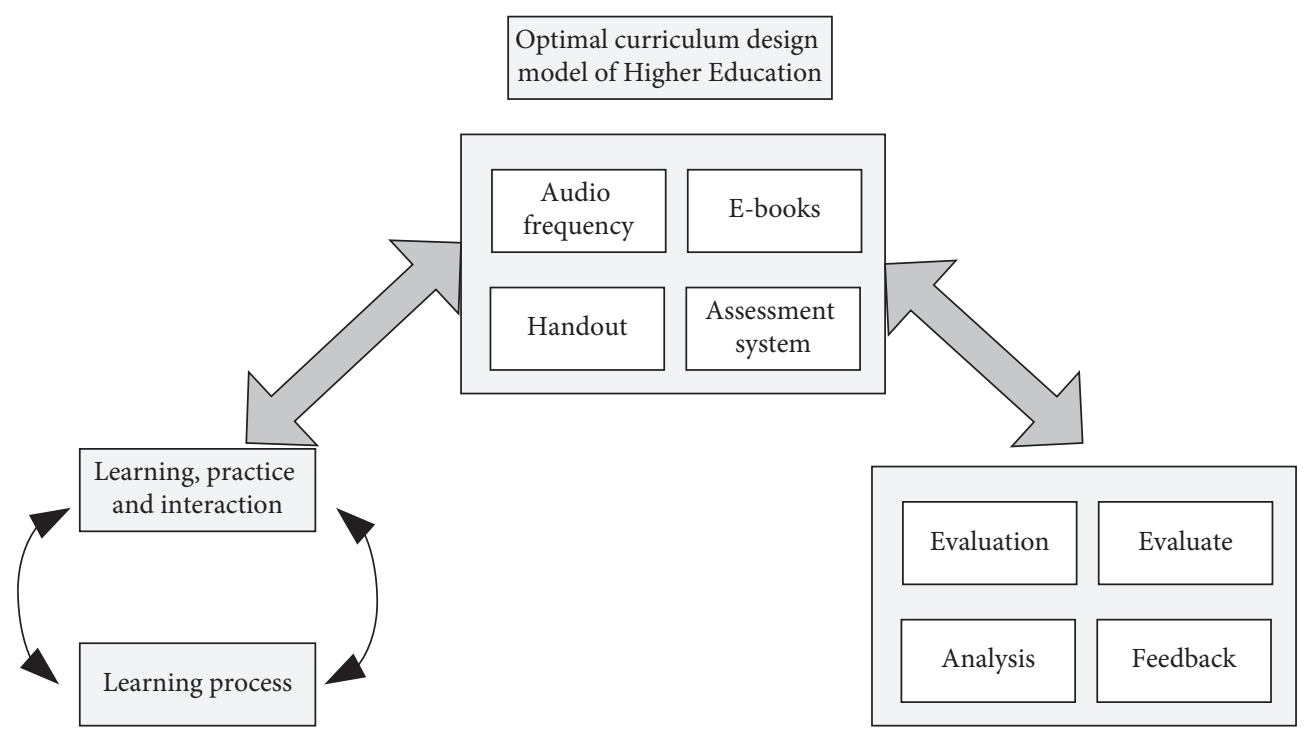

FIGURE 1: Schematic diagram of optimal curriculum design model of higher education.

represents the performance quantitative value of higher education resource evaluation and decision-making in combination with cognitive theory, with different quantitative values of evaluation and decision-making due to differences:

$$
O_{h}=\sum_{j=1}^{n} \frac{s \times \varepsilon_{f g}}{x}+\sum_{j=1}^{n} \frac{c_{k h} \times v_{s}}{x} .
$$

In the formula, $c_{k h}$ represents the conventional performance of higher education resource evaluation and decision-making, and $v_{s}$ represents the adaptability of different higher education resources; then the probability of higher education resource evaluation and decision-making with different characteristics is calculated by the following formula:

$$
\vartheta\left(V_{y}\right)=\frac{\mu_{b} \times \kappa_{r}}{J_{\alpha}^{\prime}} c_{k h} O_{h}
$$

In the formula, $\kappa_{r}$ represents the current situation of college students' resource evaluation and decision-making at this stage, and $\mu_{b}$ represents the corresponding change characteristics of college students' higher education resources with different characteristics. When the following conditions are met, the data distribution diagram of evaluation and decision-making influencing factors in the source and target fields of higher education resources is shown in Figure 2.

In Figure $2, h_{1}$ and $h_{2}$ are the factor classifiers of the source domain and target domain of higher education resources. From Figure 1, it can be analyzed that the independent variable factor distribution of the target domain is more uniform and reasonable than that of the source domain. Assuming that $h_{r}$ is the feature of educational resources, $\iota(i)$ is the attribute of educational resources, $i$ is the attribute vector of influencing factors of higher education resources evaluation and decisionmaking, and $F^{\star}$ is the selected feature, the main

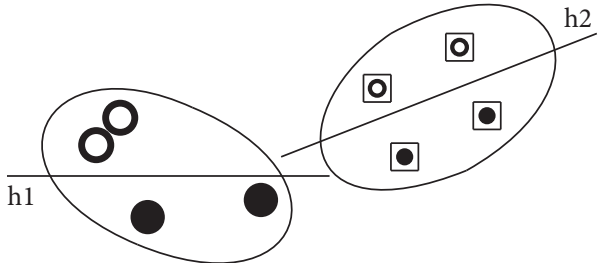

FIGURE 2: Change diagram of influencing factors of evaluation and decision-making in source and target areas of higher education resources.

impression factors formed by higher education resources evaluation and decision-making are extracted by the following formula:

$$
K_{\varsigma}=\frac{h_{r} \times z_{o}}{\tau_{m}} \times \frac{F^{\star}}{\iota(i) * a_{d}} .
$$

In the formula, $z_{o}$ represents the time required for educational resource evaluation and decision-making, $\tau_{m}$ is the failure probability of educational resource evaluation and decision-making, and $a_{d}$ is the cycle of educational resource evaluation and decision-making.

\subsection{Evaluation and Decision Analysis of Higher Education} Resources. In the process of analyzing higher education resource evaluation and decision-making, based on the determined influencing factors of higher education resource evaluation and decision-making, combined with factor analysis theory, this paper gives the positive and negative correlation between higher education resource evaluation and decision-making, obtains the method coefficient of cognitive conflict on higher education resources, and calculates the number of basic systems of influencing factors on higher education resource evaluation and decision-making. Based on the ranking results of the method quantity of educational resource evaluation and decision-making, the 
analysis of higher education resource evaluation and decision-making is realized. The specific process is as follows.

Assuming that $B_{e}$ is the correlation coefficient between the speed of higher education resource evaluation and decision-making and influencing factors, $D_{r}$ is the characteristics of higher education resources, and $M^{\omega}$ is the intermediary effect of influencing factors on higher education resource evaluation and decision-making and is the defined value of intermediary effect, then the positive and negative correlation between influencing factors and higher education resource evaluation and decision-making are given by formulae (20) and (21):

$$
\begin{aligned}
L_{k e} & =\sum_{m=1}^{i} \frac{D_{r} \times M^{\omega}}{B_{e}} \times W(v e) o_{i l}, \\
f g_{o} & =\frac{M^{\omega} \times h_{e r}}{D_{r}} * o_{i l} .
\end{aligned}
$$

In the formula, $W(v e)$ is the maximum difference between higher education resource evaluation and decisionmaking process, $p$ is the number of method effect attributes, $o_{i l}$ is the interaction between influencing factors and higher education resource evaluation and decision-making, $h_{e r}$ is the overall stress of impression factors on higher education resource evaluation and decision-making, and the method coefficient of influencing factors on higher education resource evaluation and decision-making is obtained by

$$
A_{m i}=\sum_{m=1}^{i} \frac{W(v e) \times L_{k e}}{\lambda_{(\phi)}} \times K_{\varsigma} .
$$

In the formula, $\lambda_{(\phi)}$ represents the interdependence between influencing factors and higher education resource evaluation and decision-making.

Based on the method results of the above influencing factors on the evaluation and decision-making of higher education resources, the influence quantity of higher education resources evaluation and decision-making is given by

$$
T(\theta)=\frac{\lambda_{(\phi)} \times \sigma_{i h}}{K_{\varsigma}} \times A_{m i} * \ell_{i h} .
$$

In the formula, $\theta$ represents the number of attribute categories, $\sigma_{i h}$ represents the adjustment between higher education resource evaluation and decision-making and the change of influencing factors, $\zeta_{f}$ and $\ell_{i h}$, respectively, represent the role and characteristics of higher education resource evaluation and decision-making, and the analysis of higher education resource evaluation and decision-making is based on the ranking results of education resource evaluation and decision-making quantity:

$$
G_{k}=\sum_{m=1}^{i}\left[\frac{\sigma_{i h} \times T(\theta)}{\zeta_{f}}\left(V a \times m r_{e}\right) \ell_{i h}\right]
$$

In the formula, $V a$ is the level of higher education resources and $m r_{e}$ is the nonlinear relationship between influencing factors and higher education resource evaluation and decision-making.

\section{Experimental Analysis}

In order to prove the effectiveness of higher education evaluation and decision-making research based on data mining, experiments are needed. We build an experimental simulation platform for higher education evaluation and decision-making under data mining under the environment of Hadoop. The experimental data are taken from 10 million big data in different categories of education fields in Nutch engine database to verify the overall effectiveness and feasibility of higher education evaluation and decision-making based on data mining. Table 1 shows the relationship between data quantity, evaluation, and decision-making time in higher education evaluation and decision-making methods under data mining.

Table 1 shows the higher education evaluation and decision-making method based on data mining. In the process of big data mining, with the increase of the number of big data, the time used for evaluation and decisionmaking also increases, but the time difference is small, which shows that the method proposed in this paper has a large amount of evaluation and decision-making data and fast speed. Table 2 shows the relationship between the number of higher education evaluation and decision-making and the storage space occupied by evaluation and decision-making under data mining.

Table 2 describes the relationship between the number and storage space of higher education evaluation and decision-making under data mining. It can be seen that the increase in the number of research methods does not have much impact on the storage space occupied by them, which proves that the evaluation and decision-making of higher education evaluation and decision-making methods under data mining are orderly and feasible. Figure 3 shows the impact of the specified threshold $T$ of higher education evaluation and decision-making dispersion under data mining on the evaluation and decision-making error when it is $1.3-1.4$.

Figure 3 shows the specified threshold of higher education evaluation and decision-making dispersion under data mining. When $T$ is $1.3-1.4$, the fluctuation of evaluation and decision-making error rate is relatively flat. The specified threshold of evaluation and decision-making dispersion can stably control the evaluation and decision-making error rate below 7\%. This is mainly because using the method proposed in this paper, the centroid spacing of multiple categories of higher education resources under data mining and the specified threshold of higher education resource evaluation and decision-making dispersion are combined into one class. The new category can improve the efficiency and accuracy of higher education resource evaluation and decision-making under data mining, so as to reduce the error of higher education resource evaluation and decisionmaking. Figure 4 shows the evaluation and decision accuracy of higher education resources under data mining.

It can be clearly observed from Figure 4 that the accuracy of higher education evaluation and decision-making under data mining changes with time. The longer the time, the greater the accuracy of higher education evaluation and 
TABLE 1: Relationship between data volume and evaluation and decision-making time.

\begin{tabular}{lc}
\hline Data quantity (ten thousand) & Evaluation and decision time (S) \\
\hline 100 & 5 \\
300 & 8 \\
500 & 11 \\
700 & 13 \\
900 & 15 \\
\hline
\end{tabular}

TABLe 2: Relationship between evaluation and decision quantity and storage space occupied.

\begin{tabular}{lc}
\hline $\begin{array}{l}\text { Number of evaluations and } \\
\text { decisions(piece) }\end{array}$ & $\begin{array}{c}\text { Storage space occupied } \\
(\mathrm{g})\end{array}$ \\
\hline 50 & 1 \\
100 & 3 \\
150 & 4 \\
200 & 6 \\
250 & 8 \\
\hline
\end{tabular}

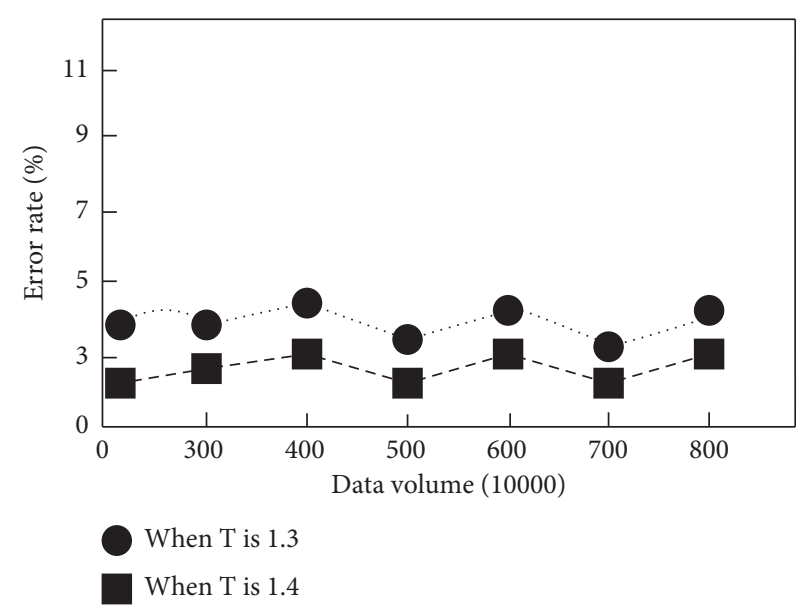

FIGURE 3: Error rate of higher education evaluation and decisionmaking.

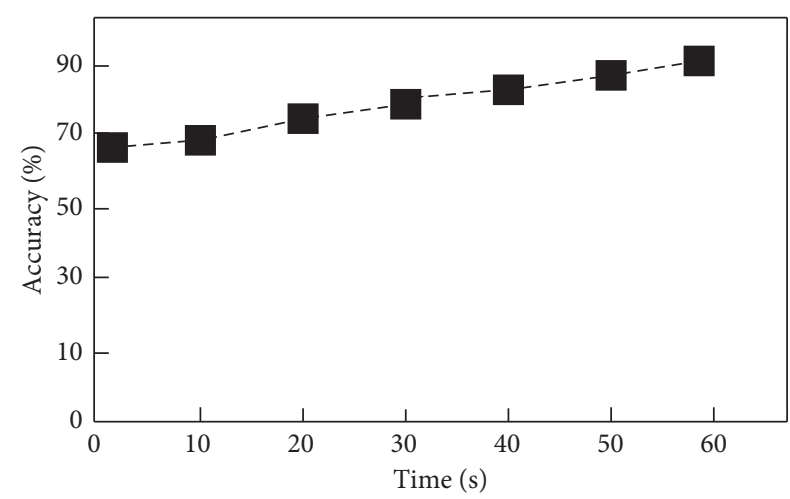

FIgURE 4: Higher education evaluation and decision accuracy.

decision-making, and it has been guaranteed to the greatest extent. It further verifies the overall effectiveness of the higher education evaluation and decision-making method based on data mining.

\section{Conclusion and Future Prospect}

The higher education evaluation and decision-making method based on data mining can not only efficiently carry out intelligent parallel evaluation and decision-making, but also make the big data evaluation and decision-making of all school information become a tool for evaluation feedback when applied to the education platform. From the big data evaluation and decision-making analysis of the school, school managers and teachers can understand the blind spots of students' knowledge, and according to the blind spots of students' knowledge, personalized teaching is carried out for students, which further improves the teaching level.

There is still much room for optimization and improvement in design analysis and research experiments, which is mainly reflected in the following aspects:

(1) In the prediction and classification evaluation of educational resource data, in the subsequent work research, we need to collect more and more complete educational resource data to improve the prediction model and classification evaluation model and find the optimal parameters to improve the accuracy of the model output data.

(2) In the balanced allocation of regional educational resources, in the subsequent work, because the current number of educational resources in each district and county can not be changed, more experiments and improvements can only be carried out in the particle swarm optimization algorithm, so as to improve the accuracy of balanced allocation of educational resources.

(3) In the research on big data visualization of educational resources, more time needs to be spent to sort out the links between data. At the same time, when visualizing the data relationship, we can consult more people's opinions and suggestions and consider the ideas of different users, so as to improve the display mode of data visualization and enable more people to obtain the hidden value behind the data.

\section{Data Availability}

The data used to support the findings of this study are available from the corresponding author upon request.

\section{Conflicts of Interest}

The author declares that he has no conflicts of interest.

\section{References}

[1] R. A. Oude Vrielink, E. A. Jansen, E. W. Hans, and J. Van Hillegersberg, "Practices in timetabling in higher education institutions: a systematic review," Annals of Operations Research, vol. 275, no. 1, pp. 145-160, 2019.

[2] V. Kourgiozou, A. Commin, M. Dowson, D. Rovas, and D. Mumovic, "Scalable pathways to net zero carbon in the UK higher education sector: a systematic review of smart energy 
systems in university campuses," Renewable and Sustainable Energy Reviews, vol. 147, Article ID 111234, 2021.

[3] V. Venkatraj and M. K. Dixit, "Life cycle embodied energy analysis of higher education buildings: a comparison between different LCI methodologies," Renewable and Sustainable Energy Reviews, vol. 144, Article ID 110957, 2021.

[4] J. S. Torrecilla, B. Gutiérrez-de-Rozas, and J. C. Cancilla, "Thinking-based learning at higher education levels: implementation and outcomes within a chemical engineering class," Journal of Chemical Education, vol. 98, no. 3, pp. 774-781, 2021.

[5] C. Romero and S. Ventura, "Data mining in education," Wiley Interdisciplinary Reviews: Data Mining and Knowledge Discovery, vol. 3, no. 1, pp. 12-27, 2013.

[6] K. R. Koedinger, S. D’Mello, E. A. McLaughlin, Z. A. Pardos, and C. P. Rosé, "Data mining and education," WIREs Cognitive Science, vol. 6, no. 4, pp. 333-353, 2015.

[7] F. Aman, A. Rauf, R. Ali, F. Iqbal, and A. M. Khattak, "A predictive model for predicting students academic performance," in Proceedings of the 2019 10th International Conference on Information, Intelligence, Systems and Applications (IISA), pp. 1-4, IEEE, Patras, Greece, July 2019.

[8] Y. Nieto, V. García-Díaz, C. Montenegro, and R. G. Crespo, "Supporting academic decision making at higher educational institutions using machine learning-based algorithms," Soft Computing, vol. 23, no. 12, pp. 4145-4153, 2019.

[9] V. V. Forrester, "School management information systems: challenges to educational decision-making in the big data era," 2019, https://files.eric.ed.gov/fulltext/ED501456.pd.

[10] M. D. C. Figueiredo, J. Amendoeira, M. Rosa, R. Matos, M. Silva, and L. Gonzaga, "Quality of life of students in polytechnic higher education at the Santarem and Leiria: the impact of COVID-19," The European Journal of Public Health, vol. 31, no. 2, pp. ckab120-036, 2021.

[11] R. P. Medeiros, G. L. Ramalho, and T. P. Falcão, "A systematic literature review on teaching and learning introductory programming in higher education," IEEE Transactions on Education, vol. 62, no. 2, pp. 77-90, 2018.

[12] S. A. Thekdi and T. Aven, "Risk science in higher education: the current and future role of risk science in the university curriculum. risk analysis," 2021, https://onlinelibrary.wiley. com/doi/10.1111/risa.13748.

[13] F. J. d. O. Araújo, L. S. A. de Lima, P. I. M. Cidade, C. B. Nobre, and M. L. R. Neto, "Impact of Sars-Cov-2 and its reverberation in global higher education and mental health," Psychiatry Research, vol. 288, Article ID 112977, 2020.

[14] T. P. Falco, "A systematic literature review on teaching and learning introductory programming in higher education," IEEE Transactions on Education, vol. 62, no. 6, pp. 77-90, 2019.

[15] S. Freeman, T.-v. Nguyen, J. Beliveau et al., "COVID-19 response strategies at large institutes of higher education in the United States: a landscape analysis, Fall 2020," Journal of Adolescent Health, vol. 68, no. 4, pp. 683-685, 2021.

[16] J. Johnes and S. Virmani, "Chief executive pay in UK higher education: the role of university performance," Annals of Operations Research, vol. 288, no. 2, pp. 547-576, 2020.

[17] J. G. Hardy, S. Sdepanian, A. F. Stowell et al., "Potential for chemistry in multidisciplinary, interdisciplinary, and transdisciplinary teaching activities in higher education," Journal of Chemical Education, vol. 98, no. 4, pp. 1124-1145, 2021. 